\title{
A multidisciplinary approach to the oral rehabilitation with dental implants: A clinical report
}

\author{
Celso Renato de S. Resende, Heraldo Elias S. dos Santos, Carlos Nelson Elias* \\ Department of Materials Science, Instituto Militar de Engenharia, Rio de Janeiro, Brazil
}

Email address:

elias@ime.eb.br (C. N. Elias)

\section{To cite this article:}

Celso Renato de S. Resende, Heraldo Elias S. dos Santos, Carlos Nelson Elias. A Multidisciplinary Approach to the Oral Rehabilitation with Dental Implants: A Clinical Report. Journal of Surgery. Vol. 1, No. 4, 2013, pp. 46-50. doi: 10.11648/j.js.20130104.11

\begin{abstract}
The oral rehabilitation treatment aims to return esthetics, function, comfort, phonetics and health to the patient. Oral treatment requires careful planning, according to the biological and mechanical principles that rule contemporary odontology. Complex clinical cases of oral rehabilitation require interdisciplinary treatment with the need for orthodontic treatment, endodontics, implants and prosthetics. This work describes a multidisciplinary clinical case of oral rehabilitation involving orthodontics, endodontics, periodontics, surgery, implant insertion and aesthetic restorative dentistry, with an implant-supported prosthesis approach.
\end{abstract}

Keywords: Dental Implants, Orthodontic Treatment, Oral Dentistry

\section{Introduction}

The aim of oral rehabilitation treatment extends to more than just the replacement of damaged or lost teeth. Rehabilitating patient with complex dental conditions means to restore aesthetics, function, comfort, phonetics and health, considering endodontic, periodontal, restorative, musculoskeletal and psychosocial factors [1].

A multidisciplinary treatment, judiciously executed according to a well-designed plan, which complies with the mechanical and biological principles, favors the achievement of success in oral rehabilitation therapy $[2,3]$.

The high rates of success and predictability obtained with dental implants have been increasing in treatments ranging from the simplest to the most complex oral rehabilitations [4, 5].

The "RM Bridge" was developed by Dr. Reginaldo Migliorança to be used as a fixed and permanent rehabilitation in edentulous patients, restoring their social life, self-esteem, chewing capacity, aesthetics and longevity of their prostheses. Among its main features are: a) The beam "I" which gives great strength to the prosthesis, with reduced volume and weight, b) individualized metal-ceramic crowns c) artificial gingival tissue similar to the patient's [6].

In the multidisciplinary approach, pre-prosthetic orthodontics allows proper installation of conventional prostheses due to a better distribution of prosthetic spaces [7]. Also an appropriate endodontic and periodontal condition of the teeth involved is essential for prosthetic rehabilitation [8].

The anamnesis and supplementary exams provide fundamental information about local and systemic conditions that create a view of the goal or the final result to be achieved [5]. The treatment should be divided in interdisciplinary steps, such as the control of active diseases; teeth reposition and preventive rehabilitation [9].

Occlusal overload is considered to be the main cause of peri-implant bone loss and failure of implant prosthesis [10]. Several factors or conditions may increase the occlusal overload, such as oversized crowns, large occlusal surfaces, high cusps, unfavorable direction of occlusal forces and the effect of cantilevers [11]. Although dental implants stimulate osseointegration, they do not provide a periodontal ligament to absorb occlusal forces [10]. It is therefore important to consider the natural dentition as well as the kind of implant to be used $[11,12]$. The occlusion is based primarily on the interaction of three elements: posterior occlusal support, occlusal vertical dimension and eccentric guides [13].

In maintenance therapy, educational measures are needed to motivate the patient to control oral health through hygiene techniques and periodic consultations $[14,15]$.

The aim of the present work is to describe a multidisciplinary clinical case of oral rehabilitation involving orthodontics, endodontics, periodontics and esthetic restorative dentistry, with an implant-supported prosthesis approach. 


\section{Clinical Case}

Male patient, 60 years, complaining of severe headaches, uninterrupted tinnitus, constant vertigo and strong facial pain, dating back to long period prior to the oral rehabilitation.

On clinical examination, patient class III, with a total conventional fixed prosthodontic, with anterior pillars on natural teeth, Garbaccio type implant and laminated bilateral posterior implants. In the lower arch there was conventional fixed prosthodontic bilateral posterior and the natural anterior teeth presented themselves buccally and with diastemata. The bite was completely crossed, with strong interference in centric relation, no disocclusion guides and alteration of the occlusal vertical dimension (Fig. 1).

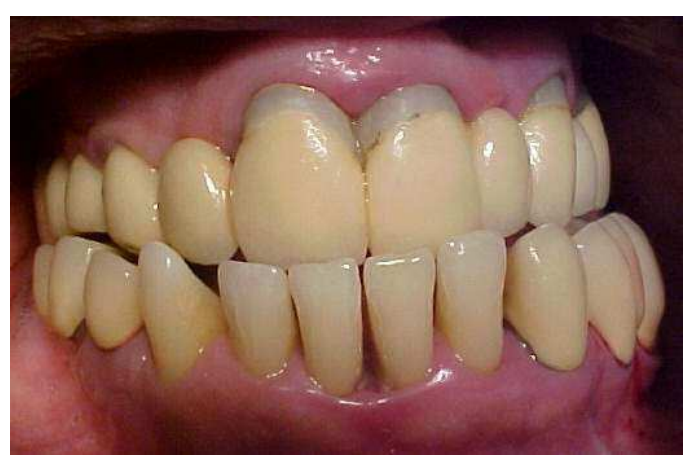

Figure 1. Patient's initial clinical condition.

Figure 2 shows the X-ray initial condition of the patient. Radiographic evaluation showed pronounced and variable loss of maxillary alveolar process, especially in posterior regions, endodontic lesions, root fractures and caries lesions. The X-ray shows a Gabaccio type implant and an old fashion laminated implant.

After orthodontic evaluation, orthognathic surgery was suggested as the first option, a possibility that was rejected by the patient.

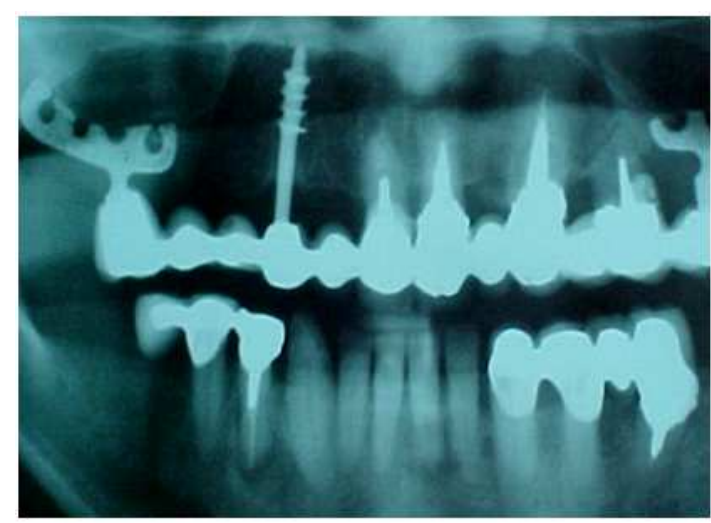

Figure 2. Patient's initial radiograph.

A basic periodontal therapy was initially established, followed by removal of the superior laminated implant, and then the remaining impaired roots and the Garbaccio implant.
Aiming at providing orthodontic anchorage for retraction of the lower anterior teeth, two mini-implants were installed in bilateral retro molar regions. Figure 3 shows the patient's orthodontic appliance, old prosthesis and removed implants.


Figure 3. Orthodontic mini-implants and device. Implant and root removed.

An immediate superior denture was installed after the extractions, following the guidance of diagnostic waxing, reestablishing the vertical dimension of the patient as well as the disocclusion guides.

With the patient without symptoms, a surgery for dental implants insertion into maxilla was done, six months after the extractions, using the temporary denture as a surgical guide. A two stage surgery was opted with a period of eight months for implant osseointegration, considering the initial lock of the implants and bone quality checked "in loco" (Fig. 4).
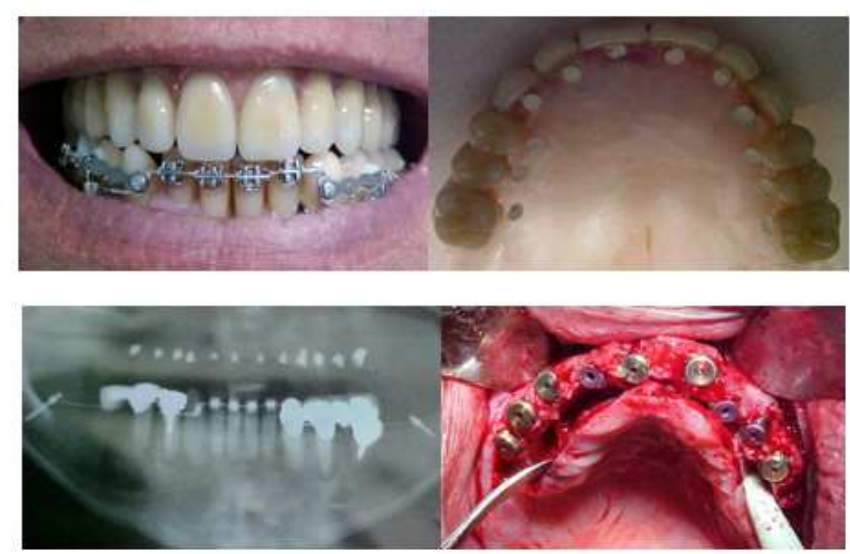

Figure 4. Superior denture, surgical guide and maxillary implants.

The lower prostheses were removed, temporary crowns installed, root canal treatments and confection of new nuclei carried out. Dental implants were installed in the posterior mandibular right and left region. A periodontal surgery was performed to correct and harmonize the lower gingival arch. Figure 5 shows the jaw after the surgery and the orthodontic appliance installed. 

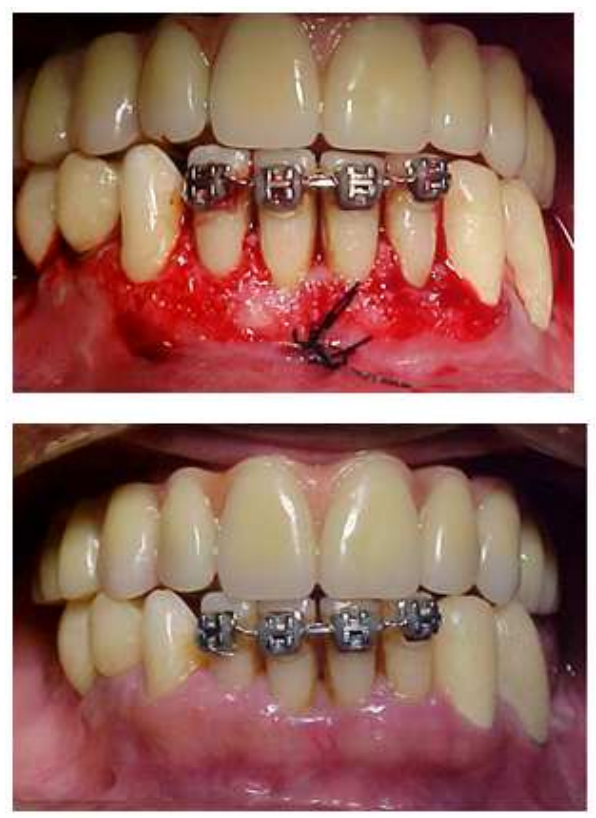

Figure 5. Lower provisional restorations and gingival arch correction.

After waiting for osseointegration of the superior implants, their transfer was conducted, as well as fabrication and testing of the metal bar, adaptation and adjustment of the upper fixed prosthesis, maintaining the same occlusal references from temporary denture that the patient was already using. Figure 6 shows the upper fixed prosthesis.

As inferior arch definitive treatment were made with metal-ceramic prosthesis on teeth and implants support, and porcelain laminated type "Empress II" on anterior teeth was used. Figure 7 shows the jaw after orthodontic treatment.
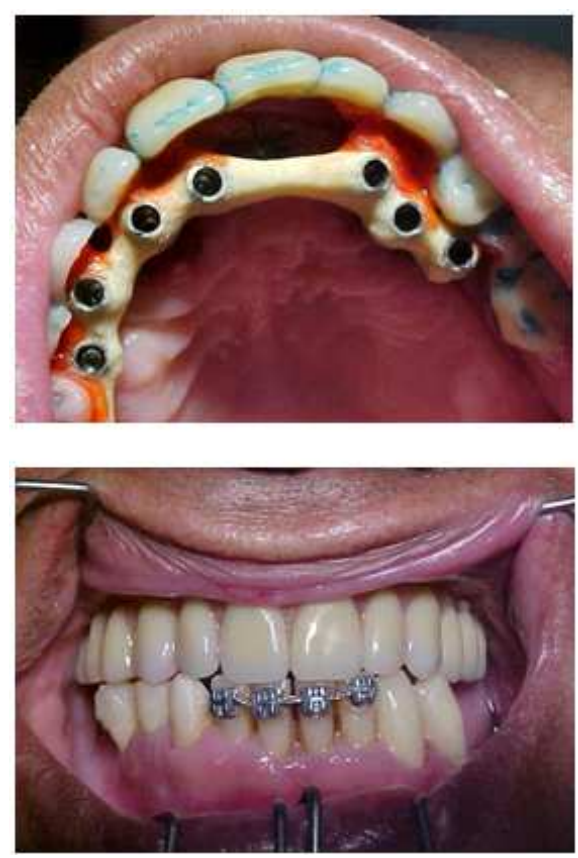

Figure 6. Temporary fixed dentistry restoration over implant.
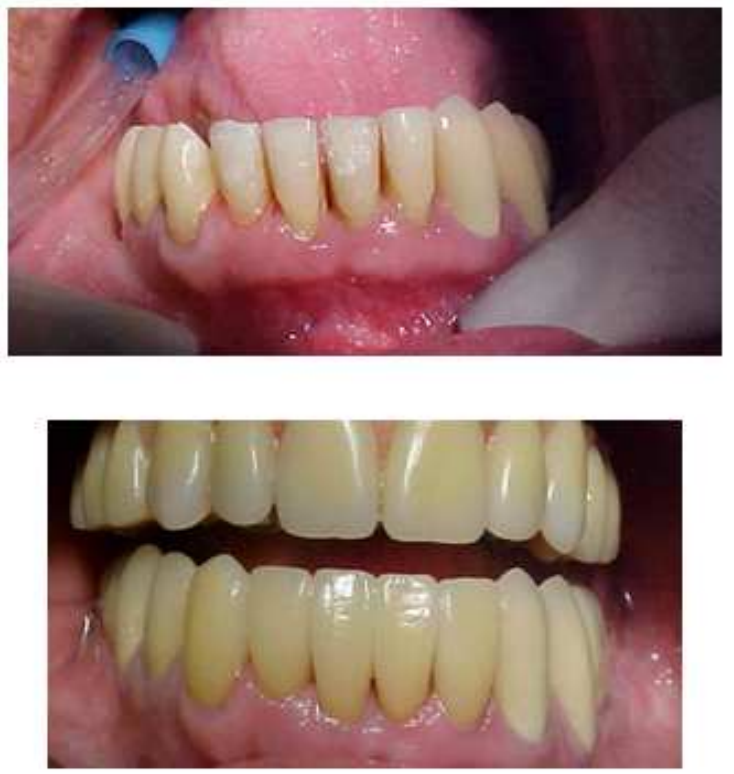

Figure 7. Lower arch definitive treatment.

A new upper arch transfer was performed and the fixed prostheses were manufactured (Fig. 8-12). Figure 8 shows the transfer registration of occlusal dimension.

Figure 9 shows the crown and the metal-ceramic prosthesis. Frameworks were fabricated on $(\mathrm{Co}-\mathrm{Cr})$ alloy and screwed into standard abutments positioned on a implant.

Figure 10 shows the patient's jaw before and after the multidisciplinary oral rehabilitation. Figure 11 shows the final patient's radiography.
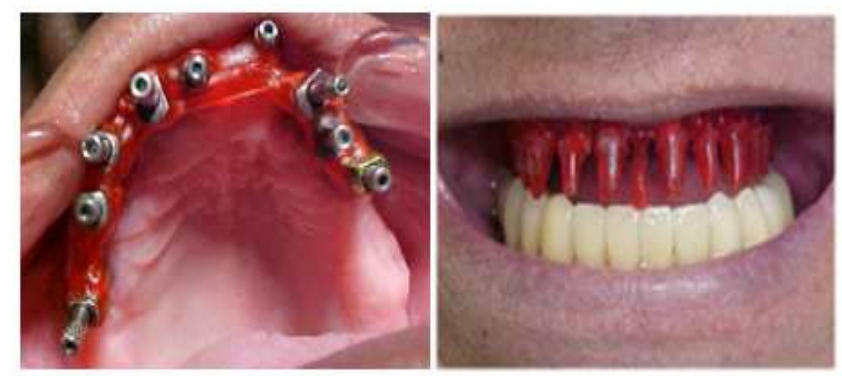

Figure 8. Transfer, registration of occlusal vertical dimension and wax try-in.

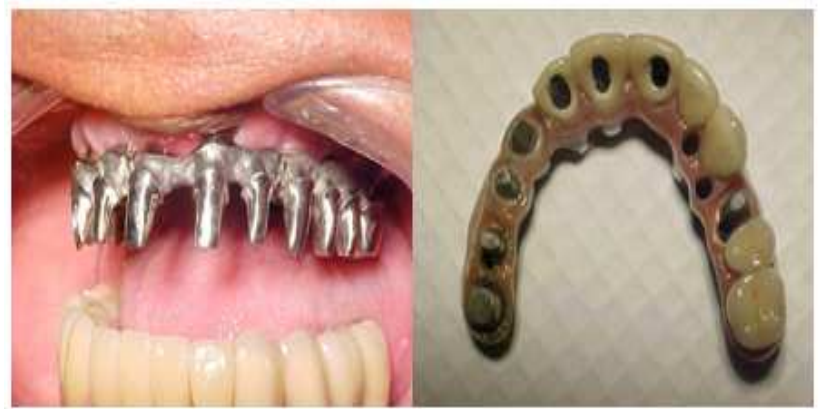

Figure 9. Metal structure and metal-ceramic prosthesis. 



Figure 10. Patient's jaw before and after multidisciplinary dentistry treatment.

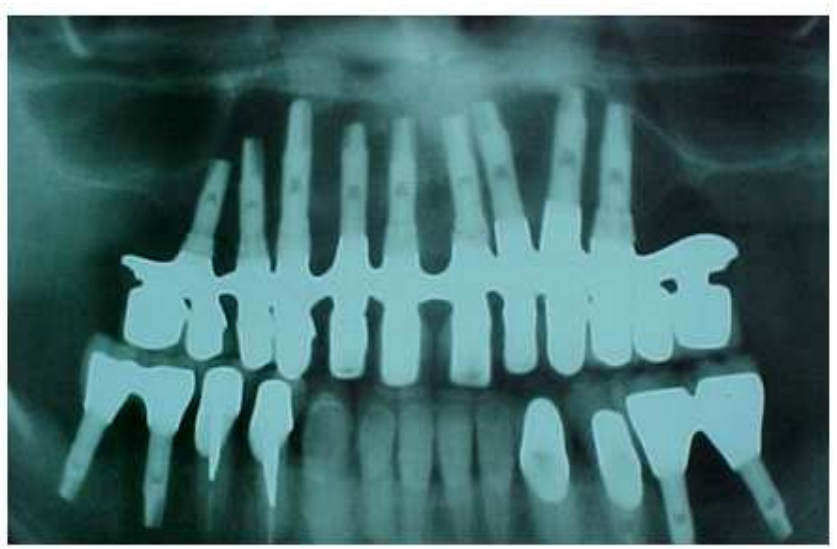

Figure 11. Pos operative radiograph made after implant surgery.

\section{Discussion}

In the present work due to the great complexity of the rehabilitation treatment, a multidisciplinary approach was planned. The present oral rehabilitation was based in the integration of various dental specialties, which allows a considerable improvement in aesthetics, function, comfort, phonetics and oral health of the patient $[9,10,13-15]$.

Other key factors to success in the present therapy of oral rehabilitation are the reverse planning and the use of diagnostic waxing. The treatment was based on literature results. These are important and necessary tools for the correct definition of the different steps required for the implementation of a rehabilitation treatment $[1,2,9,15]$.
For dentistry treatment a common choice is the indication of dental implants, which provides new options for treatment planning and execution from the simplest to the most complex oral rehabilitations. The literature reports high success rates and predictability obtained with dental implants $[2,3,8,10]$.

The treatment shown in this case report also points to the possibility of obtaining a rehabilitation of both dental arches without any prior orthognathic surgery. Obviously, in cases where the indication for such intervention is presented as a mandatory condition, such conduct should be established. Therefore, it is worth mentioning again the necessity of an appropriate, multidisciplinary planning in addition to the clarification and agreement by the patient.

\section{Conclusion}

Success in oral rehabilitation requires a careful multidisciplinary approach, based on a well-designed plan that takes into account biological and mechanical principles.

The osseointegrated implants have brought a huge contribution to oral rehabilitation, giving patients a considerable improvement in their quality of life, returning them the ability to perform a variety of functions that would be impossible using conventional prostheses, particularly the removable ones.

\section{References}

[1] A. Lodis, K.C. Kazue Yui, R. Pazinato, J.C.C. Bacigalupo, and A.B. Melo Filho, "Multidisciplinary focus to solve a clinical case", PCL: Revista Brasileira de Prótese Clínica \& Laboratorial, vol. 1, pp. 79-83, 1999.

[2] L.Q. Closs, "Orthodontics considerations in a multidisciplinary treatment approach involving implants and orthodontics", Ortodontia Gaúcha., vol. 1, pp. 13-19, 1997.

[3] C.C. Cunha, E.A.S. Coronatto, C. Bataglion, and A.C.C.I. Pereira, "Importance of myofunctional therapy in the rehabilitation of the complete denture wearer", Rev Assoc Paul Cir Dent., vol. 57, pp. 101-104, 2003.

[4] S.H.D. Moraes, G.I. Köhler, J.F.W. Köhler, N.R.W. Köhler, and V.L.B. Köhler, "Orthodontics-implants-myotherapy combined treatment - case report", JBC J Bras Odontol Clín., vol 1, pp. 21-24, 1997.

[5] D. Wismeijer, M.A.J.V. Waas, and W. Kalk, "Factors to consider in selecting an occlusal concept for patients with implants in the edentulous mandible", J Prosthet Dent., vol. 74, pp. 380-384, 1995.

[6] R.M. Migliorança, R.T. Nagahisa, T. Mayo, R.B.S. Viterbo, A.R. Coppedê, H.O. Pinto, et al. "RM Bridge: a new rehabilitation prosthetic approach", ImplantNews, vol. 4, pp. 131-137, 2007.

[7] K.M.D. Valle, A. Pinzan, S.C. Kano, G. Bonfante, and A.L.D. Valle, "Implants and orthodontics - a multidisciplinary treatment", Rev Clin Ortodon Dental Press., vol. 1, pp. 37-42, 2003. 
[8] G.D. Greco, V. Costa, A.C.D.L. Greco, W.M.S. Rocha, and M.D. Lanza, "The importance of multidisciplinary planning in oral rehabilitation treatment', ImplantNews, vol. 6, pp. 57-64, 2009.

[9] C.A.L.P. Câmara and D.M. Fonseca, "Oral recovering integrated treatment in adult patient", Rev Clin Ortodon Dental Press, vol. 3, pp. 83-90, 2004

[10] Y. Kim, "Occlusal considerations in implant therapy: clinical guidelines with biomechanical rationale", Clin Oral Implant Res., vol. 16, pp. 26-35, 2005.

[11] B. Rilo, J.L. da Silva, M.J. Mora, and U. Santana, "Guidelines for occlusion strategy in implant-borne prostheses", Int Dent J, vol. 58, pp. 139-145, 2008.
[12] H. Zanetti, A. Fagá Júnior, and C.K.H. Shimizu, "The importance of occlusion in fixed partial prothesis associated with implants", Rev Paul Odontol., vol. 21, pp. 10-14, 1999.

[13] M.D. Gross, "Occlusion in implant dentistry. A review of the literature of prosthetic determinants and current concepts", Australian Dental Journal, vol. 53, pp. 60-68, 2008.

[14] I.C. Araújo, M.V.A. Araújo, R.G. Rocha, F.E.G. Perez, C.B. Melo, and R.F.F. Barroso, "Integrated clinical planning - oral health maintenance phase”, JBC J Bras Clin Odontol Integr., vol. 10, pp. 183-187, 2006.

[15] W.C.C. Bonachela, C. Costa, P.H.O. Rossetti, and R. Freitas, "Evaluation of satisfaction degree in patients with fixed partial dentures relative to the clinical and radiographics results", Rev Bras Odontol., vol. 56, pp. 153-159, 1999. 